\title{
Mediación, un método para solucionar conflictos en instituciones de salud pública
}

\section{Aurora Kristell Frías López}

Paz y justicia social son dos principios que dan origen y sostienen el proceso de mediación, el cual es un método creado para la solución de conflictos alejados de la violencia y cobijados por el respeto, la tolerancia, la comunicación y la equidad.

Rosa Cornelio Landero, abogada y especialista en administración pública, en su libro Mediación, un método para solucionar conflictos en instituciones de salud pública bajo el sello de la editorial Porrúa (2019) describe el papel que tiene este método alternativo de solución de controversias en cualquier dimensión social. Puntualiza la función que ejerce el proceso de mediación en una sociedad en la que permea un descontento social y un hilo de frustraciones personales y colectivas entre los miembros de la misma.

La autora describe las formas de abordar un conflicto aplicando el método de la mediación, cuyas herramientas con las que funciona son el diálogo, la comunicación, la neutralidad, la imparcialidad y la escucha activa, que son a la vez cualidades encaminadas a la generación de atmósferas de paz y respeto.

Cornelio Landero logra dilucidar el papel que

Fecha de recepción: 27 de julio de 2020. Fecha de aceptación: 4 de febrero de 2021.

Cornelio Landero, Rosa (2019). Mediación, un método para solucionar conflictos en instituciones de salud pública. Ciudad de México: Porrúa. 
podría ejercer la mediación como parte de una justicia alternativa en las instituciones públicas en México, propiamente las vinculadas al servicio de salud, si se ejerciera formalmente este método para solucionar los conflictos generados interna y externamente en las instancias descritas.

La autora hace esta propuesta partiendo de la experiencia de las instituciones públicas de salud en su terruño: Tabasco. Y lo hace desde un análisis de la situación actual de la concepción de controversias en las organizaciones públicas de salud. Para ello analiza cuatro nosocomios de la capital tabasqueña desde los basamentos de comportamiento organizacional y sus consecuencias al interior como al exterior de la instancia.

El lector se encuentra con un panorama de esperanza y alcance de la paz en medio de tiempos violentos e inseguros para el ciudadano y con la posibilidad de tener acceso a una justicia social en tiempos de desesperanza, pobreza y desconfianza. Esto es posible con el proceso de la mediación.

Esta obra, derivada de un proceso de investigación a lo largo de dos años, plantea la aplicación de la mediación dentro de las organizaciones públicas de salud en la entidad y la propuesta de replicarla en el panorama nacional ofreciendo, con ello, la posibilidad de fomentar la confianza y el reconocimiento hacia todas las personas que participan en ella, desde los servidores públicos hasta los pacientes o derechohabientes, además que se garantiza su derecho humano de acceso a la salud y a la equidad social.

El lector que se acerque a la obra de Cornelio Landero podrá conocer, desde una perspectiva conceptual pero sobre todo didáctica, la función de este - aún poco conocido y aplicado- método alternativo de solución de conflictos; también podrá aprender los marcos legales y las naciones en las que ya se ha aplicado con mayor injerencia y alcances. Por lo que es un libro muy pertinente para los profesiona- 
les de las disciplinas ligadas a la mediación: el derecho, la antropología, la psicología, la sociología y la comunicación.

La obra está estructurada en dos partes. Una encaminada a las bases conceptuales y la segunda a los fundamentos originados en la práctica. Contiene seis capítulos que abordan con precisión los elementos que constituyen el método de la mediación, incluyendo el panorama experiencial de Cornelio Landero.

La primera parte, titulada "La mediación y gestión de conflictos: en busca de la paz y la justicia en las organizaciones", se integra de tres capítulos con seis núcleos temáticos en los que se describen las principales características, componentes y corrientes de la mediación. También explica, de manera precisa, las estructuras de organización de las instituciones públicas de salud, así como el derecho constitucional de los mexicanos al acceso a los servicios de salud.

Pero Cornelio Landero realiza una investigación profunda desde el marco legal internacional acerca de los organismos mundiales que se han vinculado a la generación de preceptos e iniciativas en búsqueda de la paz, además de las que se relacionan con las organizaciones, propiamente las públicas para conocer los preceptos y normas.

En el primer capítulo, nombrado "La mediación y sus principales características", se describen los medios alternos de solución de conflictos a partir de la legislación vigente en México. Estos mecanismos alternos son una posibilidad para transformar los problemas en soluciones, pues de manera inevitable éstos suelen generarse entre los individuos, los grupos y las instituciones, ya sean públicas o privadas.

En esos mecanismos destaca la mediación, ya que es un método de la justicia alternativa cuya característica es que las partes del conflicto no tienen que comparecer forzosamente ante alguna autoridad jurisdiccional para resolverlo mediante algún juez, sino sólo se requiere de la 
simple voluntad de las partes bajo la guía de un profesional llamado mediador.

En el capítulo dos, denominado "Instituciones de salud pública y derecho a la salud", la autora nos lleva por un recorrido profundo para conocer el papel que tienen las instancias derivadas del gobierno y su responsabilidad con el Estado del bienestar para sus gobernados. Se detiene y profundiza en las instancias de salud pública; desde ahí aborda, primero el derecho a la salud y la responsabilidad del Estado para garantizarla a todos los ciudadanos con equidad y satisfacción a los mismos. Pero además de describir la función del Estado, que es la de garantizar que todos tengan acceso a la atención médica, también efectúa un acercamiento a la realidad desde esa perspectiva pública.

En el tercer capítulo, que se titula "Organizaciones: la paz institucional y la gestión de los conflictos", la autora aborda la función del comportamiento organizacional tomando como eje central la gestión de los conflictos, partiendo de mecanismos alternativos de solución.

En este apartado ofrece a los lectores una exhausta explicación de los diferentes estilos, comportamientos y conductas que reflejan los miembros de la organización. Y se detiene en las de carácter público como su principal temática.

La segunda parte del libro, denominada "Los conflictos en las instituciones de salud: su actualidad y trascendencia", abarca los tres capítulos restantes. En este apartado se exponen los tipos de conflictos en las organizaciones, además de las características y las repercusiones. En esta parte el lector podrá acercarse a los diferentes tipos de conflictos, con sus respectivas actitudes.

En el capítulo cuatro, cuyo título es "Tipología de conflictos en las organizaciones y sus efectos", se incluyen los diferentes efectos de los conflictos, partiendo de los tipos; además la autora nos lleva en un recorrido por los elemen- 
tos que caracterizan a cada uno, así como las formas más factibles de acabar con ellos.

Ahí aparecen los conflictos internos, que a su vez pueden ser positivos o negativos, dependiendo de cómo se establezca la comunicación en relación con los valores, las metas y las aspiraciones.

En la sociedad contemporánea, dentro del contexto organizacional los conflictos se consideran como un fenómeno normal, inevitable y esperado. Cornelio Landero propone que para acabar con el conflicto debe comprenderse y atenderse como una oportunidad de transformación, si se maneja en una forma productiva y efectiva para hacer de la experiencia negativa una positiva.

Si el conflicto no se atiende, entonces corre el riesgo de convertirse en un prejuicio para la institución, así que hay que detectarlos y tratarlos oportunamente, antes de que se vuelvan una pesada carga; además -apunta la autoracada persona posee una forma particular de enfrentar los conflictos, pero el momento y el espacio en que tienen lugar también son condicionantes.

En el capítulo cinco, denominado "Enfoque proactivo frente al conflicto", la autora señala que las organizaciones deben anticiparse a los problemas o necesidades que puedan detonar conflictos. Dice Cornelio Landero que ser proactivo es trabajar con diligencia empleando una energía positiva, en donde las instancias con los conflictos difíciles ofrezcan soluciones constructivas, de cambio o de transformación para el crecimiento de las mismas.

Además define que este enfoque proactivo permitirá que un conflicto bien gestionado sea una fuente de aprendizaje y un marco de apertura de nuevas posibilidades.

Otro de los elementos que cita la autora en este apartado es la violencia existente en las organizaciones, también conocida como violencia estructural, cuya peculiaridad es su dificultad para reconocerla. 
La autora nos explica con precisión que la violencia en la organización altera el trabajo en equipo y obstaculiza el alcance de sus objetivos. Argumenta que uno de los factores de la violencia en el trabajo es el medio social, ya que las organizaciones suelen estar ubicadas en contextos inseguros y con baja satisfacción para los empleados.

Es un hecho la existencia de la violencia en muchos trabajadores de servicio público, que va desde el maltrato, el acoso sexual, amenazas e intimidaciones y otras formas de violencia psíquica tales como la humillación y el menosprecio. En este capítulo aparece una distinción de los tipos de violencia que más ocurren al interior de las instituciones. Tales como el mobbing o acoso laboral, el cual es una violencia psicológica, acoso moral o acoso laboral; en otros términos, una medida de conducta agresiva que ha alcanzando niveles elevados, generando poca productividad en la organización.

Otro de los tipos de violencia es el estrés laboral, que es un proceso psicológico que se da como respuesta a presiones ambientales. Cuando un trabajador sufre de estrés, la productividad de las organizaciones disminuye. Y el síndrome de burnout que hace que los empleados sientan agotamiento físico y mental de forma progresiva; muchas veces cuando el trabajador está desmotivado para realizar sus funciones y tareas, o más bien las hace pero nadie se las reconoce, es probable que se presente un cambio de actitud.

Todos estos tipos de violencia laboral tienen como secuelas los conflictos y es ahí donde la autora propone el uso de la mediación para contrarrestar estos fenómenos negativos que existen, principalmente en las instituciones públicas.

En el capitulo sexto, el último y al que ha titulado "Prevención, gestión y solución de conflictos en las instituciones publicas de salud a través de la mediación", la autora aborda el panorama de las instituciones públicas de salud en México a partir de la experiencia de Tabasco.

\section{6}


Explica que la preocupación que origina este libro, gira en torno a la existencia cotidiana de conflictos diversos entre el personal que labora al interior de los nosocomios, lo cual se debe a las dinámicas de las funciones que realizan y los servicios.

Esta experiencia basada en la investigación de Cornelio Landero le permite proponer que la mediación es una vía que debe aplicarse para resolver problemas con el objeto de establecer acuerdos que satisfagan intereses mutuos.

La autora describe el panorama de cuatro nosocomios de la capital tabasqueña, y profundiza a través de las percepciones de los encargados de los departamentos de recursos humanos respectivos de cada instancia.

De ahí se derivaron los hallazgos planteados en este apartado, en primer lugar que las instituciones de salud pública no cuentan con métodos alternativos que permitan resolver los conflictos, considerando el impacto económico que éstos pueden generar, la existencia de los malos tratos y las ofensas entre compañeros de algunas áreas de departamentos, que se presentan cuando los empleados traspasan sus problemas personales, económicos o familiares al trabajo y se desquitan con sus colaboradores.

En esa misma ilación de hallazgos, describe cómo impactan los problemas personales en el trato y atención al público. La autora propone la aplicación de la mediación como un método de solución de conflictos, la cual es totalmente desconocida en las instituciones públicas de salud.

Rosa Cornelio Landero abre a la reflexión sobre la importancia de mediación en cualquier fase de la vida del ser humano, porque es transformativa, apela a la no violencia, hace uso de la comunicación, es dialógica, se basa en el respeto y la imparcialidad, es empática y solidaria, restaura positivamente a las partes del problema, apela a la justicia social y, principalmente, es sin duda: un instrumento generador de la paz. Sí, esa paz a la que toda sociedad aspira. 
Esa misma paz que hoy México demanda, anhela, pero sobre todo necesita. 